\title{
Detection of Osteoarthritis using Knee X-Ray Image Analyses: A Machine Vision based Approach
}

\author{
Shivanand S. Gornale \\ Associate Professor, \\ Department of Computer \\ Science, School of \\ Mathematics and Computing \\ Sciences, \\ Rani Channamma University, \\ Belgavi. Karnataka-India
}

\author{
Pooja U. Patravali \\ Research Scholar \\ Department of Computer \\ Science, School of \\ Mathematics and Computing \\ Sciences, \\ Rani Channamma University, \\ Belgavi. Karnataka-India
}

\author{
Ramesh R. Manza \\ Associate Professor, Bio- \\ Medical Image Processing \\ Laboratory, Department of CS \\ \& IT ,Dr. Babasaheb \\ Ambedkar Maratwada \\ University, Aurangabad- \\ Mharashtra-India
}

\begin{abstract}
Osteoarthritis is one of the popular causes of debility in elderly \& overweight people. Osteoarthritis is a joint disease that invades the cartilage of bigger joints like knee, hip, feet and spine. Cartilage helps the easy glide of bones \& obstructs them from rubbing each other. In Osteoarthritis cartilage is ruptured due to which bones start kneading each other with a severe pain. The scenario for the evaluation of Osteoarthritis includes clinical examination \& various medical imaging techniques. In this work the authors have used Active contour segmentation technique to segment the portion/part of the knee X-ray image to diagnosis the disease. The numerous features like Haralick, Statistical, First four moments, Texture and Shape are computed and classified using Random Forest classifier. The proposed method gives the classification accuracy rate of $87.92 \%$ which are more competitive and promising with the existing algorithms.
\end{abstract}

\section{General Terms}

Machine learning, Feature extraction algorithm, Statistical Classification, Medical imaging

\section{Keywords}

Osteoarthritis, Knee X-ray, Activecontour algorithm, Random forest.

\section{INTRODUCTION}

Medical imaging [17][18] is the process of creating visual representations of the internal structures hidden by the skin and bones. It is the technique where the interior parts of the body for clinical diagnosis and medical intervention are revealed. Medical imaging is a part of biological imaging that incorporates various imaging technologies like X-ray [19], Magnetic Resonance Imaging (MRI), Ultrasound, Computed Tomography (CT) etc [20]. Osteoarthritis is one of the most common form of arthritis disease that is seen mostly in females, overweight and elderly people [21][3][22].Osteoarthritis (OA) is a joint disease that mostly affects the cartilage [21]. Cartilage is the protective connective tissue that covers the end of bones in a joint. The normal or healthy cartilage allows easy glide of bone in the joint and prevents them from rubbing each other [23]. In Osteoarthritis the top layer of cartilage is erupted due to which the bones rub each other causing severe pain [3][4].Osteoarthritis commonly affects the joints in the knee, hip, spine and feet. There are two types of OA, Primary OA seen in aged people due to genetic reasons or aging. Secondary OA tends to show up earlier in life due to some injury, diabetes, obesity, athletics or patients with rheumatoid arthritis. The sample of normal \& affected Osteoarthritis knee image is shown below in figure 1 and 2 .

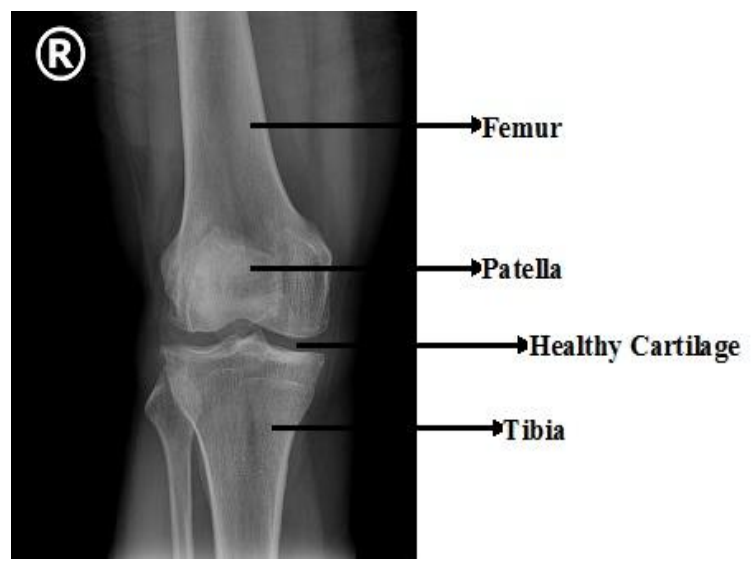

Fig 1: Normal Knee Image

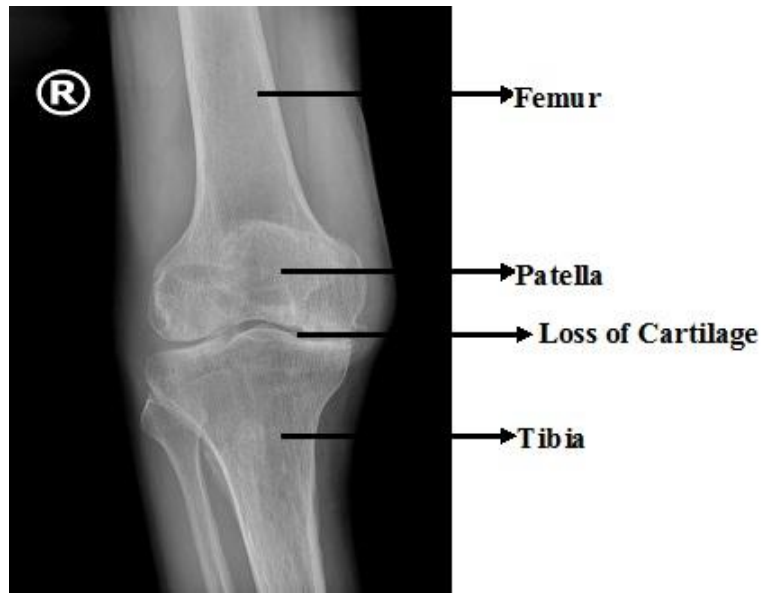

Fig 2: Osteoarthritis Knee Image

The main symptoms of $\mathrm{OA}$ are pain and difficulty in joint motion, reduced function and participation restriction, Joint stiffness in the morning or after prolonged rest. The present evaluation of $\mathrm{OA}$ is based on clinical examination, symptoms and simple radiographic assessment techniques (X-ray), MRI, CT etc. While several other methods have been proposed, Kellgren-Lawrence (KL) system is validated method of classifying individual joints into 5 grades [1]. Table below shows the different grades of OA disease. 
Table 1: KL Grading System

\begin{tabular}{|l|l|}
\hline $\begin{array}{l}\text { KL } \\
\text { Grades }\end{array}$ & \multicolumn{1}{|c|}{ OA Analysis } \\
\hline Grade 0 & No Radiographic features of OA present \\
\hline Grade 1 & Doubtful OA(narrowing of joint space) \\
\hline Grade 2 & Mild OA(definite narrowing of joint space) \\
\hline Grade 3 & Moderate OA (multiple osteophytes, sclerosis) \\
\hline Grade 4 & $\begin{array}{l}\text { Sever OA ( large osteophytes, sever sclerosis, } \\
\text { bone deformity) }\end{array}$ \\
\hline
\end{tabular}

The common X-ray findings of OA include destruction of joint cartilage; joint space is diminished between adjoining bones and bone spur formation [24]. MRI scans may be ordered when $\mathrm{x}$ - rays do not give clear reason for joint pain or when the $\mathrm{x}$ - ray suggests that other type of joint tissues can be damaged. In OA, after acquiring the Knee X-ray image, human experts need certain time to examine the knee X-ray image. The parameters used for the classification of $\mathrm{OA}$ are continues \& complex. It may be possible that the experts may exceed time limit in investigating the knee $\mathrm{x}$-ray image and may reach some other conclusion regarding the presence of OA. So to overcome these human errors Computer aided automation of knee x-ray image is required. Automation allows quick and efficient results with respect to the disease \& has significantly less errors. Sometimes knee X-ray images may not be clear during early Osteoarthritis or due to some other distortions. If the knee X-ray findings are not clear doctor suggests the patients to go for MRI. As MRI is an expensive option, common man may not afford it. The current methods used for clinical diagnosis of Osteoarthritis are not accurate enough to efficiently measure the quality \& evolution of Osteoarthritis. Thus more significant methods \& algorithms are required which are multi-factorable to access the parameters \& progression of Osteoarthritis. Therefore the authors have tried to implement semi-automated method that helps in the diagnosis of the disease to some extent.

In the rest of the paper, Section- 2 contains the review work that includes different segmentation \& feature extraction techniques used for the classification of Osteoarthritis based on knee X-ray imaging. Section-3 describes the methodology. Section-4 includes experiment analysis and lastly Section-5 has conclusion along with future scope followed by qualitative references.

\section{RELATED WORK}

As per the preceding survey, researchers have investigated various methods on the detection and analysis of Osteoarthritis using different knee images like X-ray, MRI etc. Image segmentation method has a great importance in most medical imaging applications [34]. In medical imaging the segmentation methods are classified into two basic groups: pixel based (includes thresholding, region growing region merging etc) and geometry based (includes deformable models, active contours active appearance models). Therefore to know the proper progression \& extremity of the disease medical imaging is carried out in terms of X-ray imaging or Magnetic Resonance imaging. As per the survey,

Lior Shamir et al [1], have used joint detection algorithm and feature extraction technique to analyze the knee X-ray image. The algorithm finds the joint and separates it from rest of the images. The features were computed by Zernike features, Multi-scale histograms, first four moments, Tamura texture features etc. The features extracted were classified using weighted Nearest Neighbor rule. The authors concluded that
95\% of moderate OA was differentiated from normal OA and $80 \%$ of minimal OA was differentiated from normal.

Samir K.Bandyopadhyay[2],have used edge detection algorithm, to detect edges of Knee X-ray images in osteoarthritis. The features computed were femur, tibia and patella cartilage. The authors have concluded that the proposed algorithm gives sufficiently good results and is very effective in noisy and blur images.

M.Subramonium et al[4], have used Local Binary Pattern(LBP) based classification system for the detection of OA using Knee X-ray images.The classification is achieved by computing the histograms of LBP of knee X-ray images using K- Nearest Neighbor classifier. The authors have concluded with $95.24 \%$ of accuracy in detecting normal or abnormal knee image and $97.37 \%$ of accuracy in detecting medium or worst cases.

Prafull Sharma et al [5], have used different Image segmentation algorithms on X-ray bone images. The discrete step, Watershed segmentation and Otsu's segmentation are used to analyze the abnormalities and problems associated with bone structures. The authors have concluded that discrete step algorithm provides quick and efficient results.

Bindushree $\mathbf{R}$ et al [6], have used different image processing techniques to measure the joint space width in knee $\mathrm{x}$-ray images. Different techniques used are contrast enhancement, histogram equalization, canny edge detection algorithm and thresholding for extraction \& computation of features. The authors have concluded that the Joint Space Width of the knee $\mathrm{x}$-ray image is compared with the standard Width (4.8 for women \& 5.7 for men) and that image is said to be normal case or osteoarthritis case.

Dian Pratiwi et al [7], have used artificial neural back propagation method for measuring the severity of Osteoarthritis disease. In their work the whole processing is divided into three steps, image processing, feature extraction and classification using artificial neural network process. They have concluded with $66.6 \%$ of classification rate.

Subromoniam M et al [8], have used computer aided diagnosis for the detection of OA using X-ray images. Haralick feature extraction technique for computation \& SVM classifier with the kernel functions are used for the detection $\&$ classification of OA. They have concluded that the algorithm had a sufficient good result with accuracy of $99 \%$ in the diagnosis of bone disorders caused by OA

Lilik Anifah et al [9], have used fully automated method to segment junction space area (JSA) for OA classification on impaired Knee X-ray images. In their proposed work right and left knee detection is performed using contrast limited adaptive histogram equalization (CLAHE) \& template matching is used to segment the joint space area of knee. In their work the whole method is divided into two steps, first step is to segment right \& left knee and second step is to find JSA. The first step is again divided into four experiments; first experiment uses centre of mass, second uses only templates matching, third uses CLAHE \& template matching and fourth combines all the above experiments. Similarly second step is divided into 2 experiments, first uses row sum graph \& centre of mass method \& second uses Gabor filter ,row sum graph \& centre of mass method. The results showed that experiment 4 in first step gave sufficiently good result with $100 \%$ accuracy and in second step experiment 2 gave $92.63 \%$ of accuracy for right knee and $87.37 \%$ for left knee. 
Mahima Shanker Pandey et al [10] have used automated computer assisted method to detect OA based on joint space width (JSW) of knee x-ray images. In their work image is enhanced using Gaussian low pass filter \& stretched using contrast stretch technique. The better quality image is obtained from which mean \& variance is calculated using neighboring pixel method. Lastly Euclidian distance formula is used on the binary format of the image to calculate the JSW which is compared with the normal value. They have concluded that among 80 images the success rate was up to $90 \%$ and $10 \%$ images failed as thresholding was not clear.

Jessie Thomson et al [11], have used fully automated computer aided system that identifies the outlines of the bones of knee joint of OA disease. The authors have Random Forest Regression Voting Constrained Local Model (RFCLM) and Statistical shape model for automated analysis. In their work two different classifiers are used to distinguish between the texture in knee structure of normal \& affected OA. Features computed were texture features, shape features, simple pixel features etc. The experiment showed that combining shape \& texture based classifiers resulted in $84.9 \%$ of accuracy as compared to shape alone.

Lilik Anifah et al[12], have used Contrast Limited Adaptive Histogram Equalization (CLAHE) \& template matching schemes. The Gabor kernel, row sum graph, moment methods and grey level centre of mass (GLCM) are used to get the joint space width area. The features computed were in terms of contrast, correlation, energy \& homogeneity. The classification rate of $93.8 \%$ for KL grade $0,70 \%$ for KL grade $1,4 \%$ for $\mathrm{KL}$ grade $2,10 \%$ for KL grade $3 \& 88.9 \%$ for KL grade 4 was obtained.

Tati L.Mengko et al[13], have used machine vision system for osteoarthritis assessment. In their proposed method image segmentation uses edge detection method to determine the region of joint space. The feature computed is the distance between femur \& tibia bone. The classification of normal \& affected OA is obtained from radiographic image using neural network that is later examined with the predefined diagnosis managed by the physician. They concluded with $50 \%$ sensitivity, $100 \%$ specificity \& positive predictive value $\&$ $91.84 \%$ negative predictive value.

Lior Shamir [14], have developed image analysis method that can find correlations between radiographic findings \& clinical indicators. The methods includes WND-CHRM algorithm using different feature extraction techniques (Haralick, Tamura, Shape, Statistical). The computed features are classified using weighted Nearest Neighbor scheme. They have concluded that the method demonstrated the satisfactory association between the radiographic findings \& clinical indicators.

Subromonium.M et al[15], have developed the algorithm to compute the features from digital $\mathrm{x}$-ray images using Local Ternary Pattern (LTP) and classifying them by using Support Vector Machine Classifier. $50 \mathrm{X}$-ray samples were used to evaluate the performance of the algorithm. They have concluded that the Linear \& Polynomial functions gave $91.66 \%$ of specificity \& $80 \%$ of sensitivity. RBF gave $94.59 \%$ of specificity \& $66.66 \%$ of sensitivity.

Chanda Ray et al[16], have used automatic clustering of Xray images by K-means method on large feature vector \& hierarchical technique is applied on $\mathrm{k}$-means clustering. The clustering is done on multilevel features of X-ray images such as global level, local level \& pixel level. They have concluded that the combination of k-means \& hierarchical clustering produces very high level of accuracy.

From the literature it is observed that a good number of researchers have worked on Knee X-ray imaging for detection and classification of OA using different approaches and forecasted some promising results with their own datasets. But still there is a scope for developing a robust algorithm using different parameters like age group, demographic characterization based on rural and urban people. Different robust features are required to be extracted from Knee X-ray for the detection of Osteoarthritis. The comparative analysis with promising results were made by researchers as predicted and discussed in the related work. But still there are certain algorithms \& methods which are open to lot of improvements. Thus, the authors need to develop a robust and precise algorithm that is suitable for evaluating the disease in early stage with high classification rate.

\section{PROPOSED METHEDOLOGY}

The proposed methodology below figure 5 consists of five main steps, Preprocessing, Image segmentation,Image enhancement, Feature extraction and Classification of computed features. The steps are explained in the following subsections[34].

\subsection{Image Acquisition}

Image acquisition can be designated as the action of fetching an image from some source which is further processed to get new and better image [25].

Data Set: The authors have used the input data set of 200 knees X-ray images that were collected from various hospitals and diagnostic centre. The images collected were based on various specifications like age, gender, blood group, occupation etc.

\subsection{Pre-processing}

Pre-processing highlights some important features relevant to understand the image. After collection of knee X-ray images, initially pre-processing techniques are used which are application dependent. In the work pre-processing is carried out by cropping the image to $512 \times 409$ pixels and resized to $250 \times 250$ for the proper analysis..

\subsection{Image Segmentation}

The segmentation is the process where the image is partitioned into its constituent parts or objects that can be identified individually [26]. In the work image segmentation is carried out using Active contour segmentation method. The boundaries of designated mask are used by Active contour as the initial state of the contour from where the evolution starts. The prominence and the suppressed regions of an image are segmented using active contour algorithm. The algorithm uses Chan-Vese and Edge methods to segment an image [28].The region between the femur and tibia is segmented and further processed for computation. The picture below shows the segmented region of Knee X-ray image. 


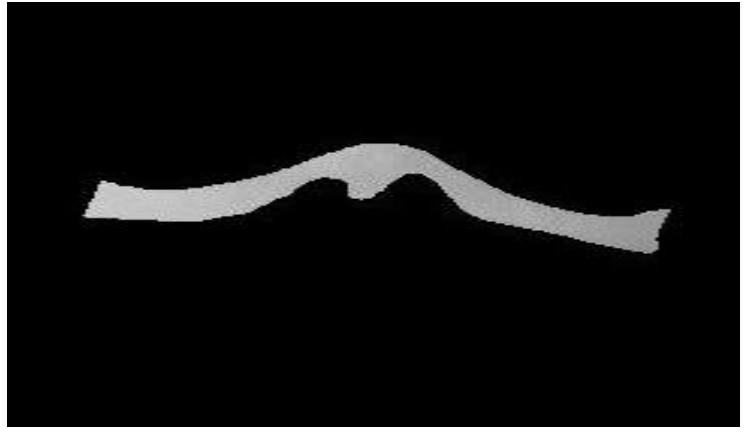

Fig 3.Segmented Image

\subsection{Image Enhancement}

Enhancement techniques are used to improve the quality of the image to great extent. The technique can improve image quality in terms of shading, linear contrast adjustment, unsharp masking, and median filtering and color [3]. In the work segmented knee X-ray images are enhanced by contrast adjustment to get better extremity of an image. The picture below (figure 4) shows the enhanced segmented region of Knee X-ray image.

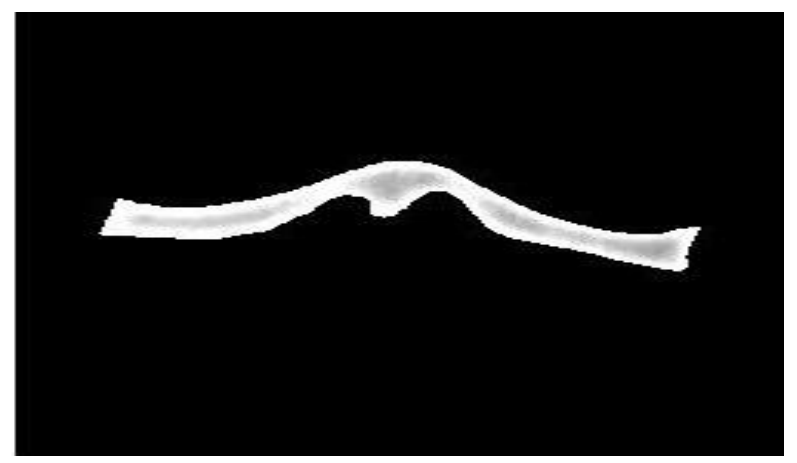

Fig 4.Enhanced Segmented Image

\subsection{Feature Extraction}

Feature Extraction [29] is one of the important modules of image processing. In this stage we compute features of segmented and enhanced image using MATLAB which results in recognition accuracy with very simple classification module. Authors have computed various features from the obtained segmented region. The features computed are:

First Four Moments: The quantitative measure of the shape with various set of points [30]. In our work the moments used are skewness, kurtosis, variance and standard deviation. The equations are as follows:

$$
\begin{array}{ll}
\text { - } & \text { Skewness }=\frac{E(x-\mu)^{2}}{\sigma^{3}} \\
\text { - } & \text { Kurtosis }=\frac{E(x-\mu)^{4}}{\sigma^{4}} \\
\text { - } & \text { Variance }=\frac{i}{N-1} \sum_{i=1}^{N}\left|A_{i}-\mu\right|^{2}
\end{array}
$$

- Standard Deviation $=\sqrt{\frac{\mathrm{i}}{\mathrm{N}-1} \sum_{\mathrm{i}=1}^{\mathrm{N}}\left|\mathrm{A}_{\mathrm{i}}-\mu\right|^{2}}$

- Statistical features: Includes assembling, formulating, determination \& clarification of data [3]. The statistical measures considered are mean, area, entropy and Euler number of images. All the statistical properties are calculated based on individual pixels. The individual pixels are determined by looking at its 2-by-2 neighborhood. The following are some features:

- Mean (M) =computes the average of an array I using mean (I (:)).

- $\quad$ Entropy $=-\operatorname{sum}(\mathrm{p} \cdot * \log 2(\mathrm{p}))$

Shape features [29]: These include measuring the similarities between shapes represented by their features [3]. The shape features are calculated based on connected components stored in contiguous and dis-contiguous regions. The following are the features computed:

- Area is the number of pixels of an image.

- Major Axis Length specifies the length of major axis of an object in pixels.

- Minor Axis Length specifies the length of minor axis of an object in pixels.

- Perimeter is the number of boundary pixels

- Equiv diameter $=\sqrt{(4 * \text { Area } / \pi)}$

- $\quad$ Eccentricity $=\frac{\lambda_{1}}{\chi_{2}}$ (Foci of ellipse/Major axis length)

- $\quad$ Euler Number=(No of objects)-(No of holes)

Haralick features[3][31]: These help in measuring the texture of the image in terms of contrast, correlation, sum of squares, sum of average, homogeneity etc. Following are some equations used

- $\quad$ Contrast $=\sum_{\mathrm{i}, \mathrm{j}}|\mathrm{i}-\mathrm{j}|^{2} \mathrm{p}(\mathrm{i}, \mathrm{j})$

- Correlation $=\sum_{\mathrm{i}, \mathrm{j}} \frac{(\mathrm{i}-\mu \mathrm{i})(\mathrm{j}-\mu \mathrm{j}) \mathrm{p}(\mathrm{i}, \mathrm{j})}{\sigma_{\mathrm{i}} \sigma_{\mathrm{j}}}$

- $\quad$ Energy $=\sum_{i, j} p(i, j)^{2}$

- Homogeneity $=\sum_{i, j} \frac{p(i, j)}{1+|i-j|}$

Texture analysis features [33]: These features calculate the local range, standard deviation and entropy of a gray scale image. All the features determine the centre element of neighborhood by floor $(($ size $(\mathrm{NHOOD})+1) / 2)$.

\subsection{Classification}

A random forest is a classifier consisting of a collection of tree structured classifiers $\{\mathrm{h}(\mathrm{x} \Theta \mathrm{k}), \mathrm{k} \square 1, \ldots\}$ where the $\{\Theta \mathrm{k}\}$ are independent identically distributed random vectors and each tree casts a unit vote for the most popular class at input $x$ [32]. 


\section{Training Phase}
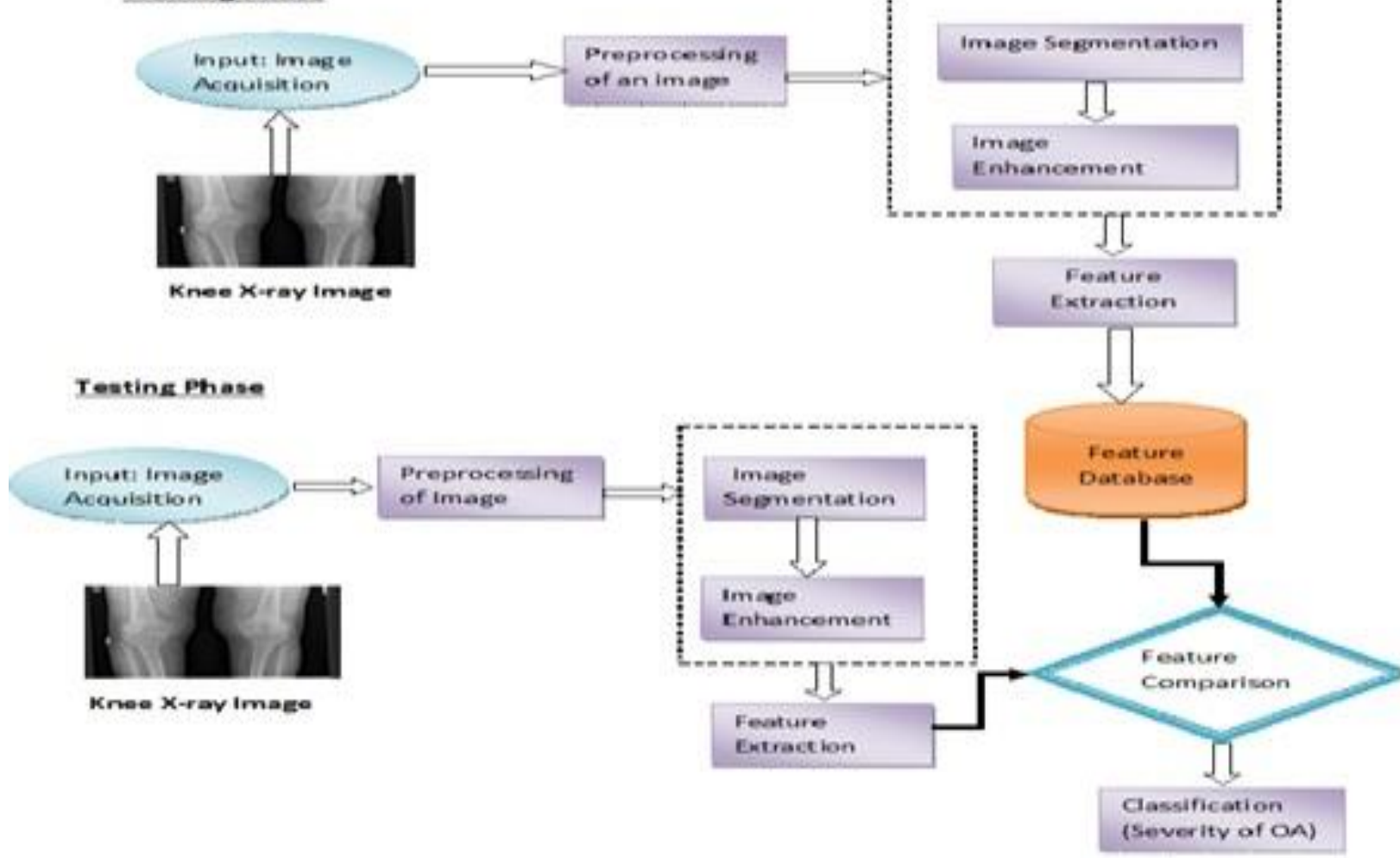

Fig 5: Block Diagram

\section{EXPERIMENT ANALYSES}

The experiment is carried out on various Knee X-ray of different age and blood groups of the rural and urban population. The experiment is conducted on 200 Knee X-ray samples. Different features were computed like Shape features, Statistical features, First-four moments, Haralick features and Texture analysis features. In the investigation $40 \%$ of training and $60 \%$ of testing was carried out for the analysis. The Random Forests algorithm was used to classify the image. The classification rate of $87.92 \%$ was demonstrated provided the given image is normal or affected by using below algorithm.

Input: Knee X-ray image.

Output: Normal or Affected Knee X-ray image.

Step-1: Pre-processing i.e. removal noise, images resize, etc and Convert it into gray-scale.

Step-2: Normalize gray scale image to size $250 \times 250$ for further analysis

Step-3: Segmentation is carried out using Active contour algorithm.

Step-4: The segmented image is enhanced using Contrast adjustment technique for further interpretation.

Step-5: The different features computed are Shape features, Statistical features, First-four moments, Haralick features and Texture analysis features.

Step-6: Lastly $40 \%$ training and $60 \%$ testing is carried out on the obtained list of features using Random Forest classifier.

Step-7 End of the algorithm.

The outcomes of the algorithm are demonstrated in the below Tables.Table- 2 gives the classification rate of individual feature set. Table- 3 gives the classification rate of merged or integrated feature set. Table- 4 gives the confusion matrix of individual feature set.

Table-2 Classification rate of individual feature set

\begin{tabular}{|l|l|l|l|}
\hline Feature Set & $\begin{array}{l}\text { Correctly } \\
\text { Classified }\end{array}$ & Misclassified & Accuracy rate \\
\hline $\begin{array}{l}\text { Texture } \\
\text { Feature Set }\end{array}$ & 123 & 84 & $59.42 \%$ \\
\hline $\begin{array}{l}\text { Haralick } \\
\text { Feature Set }\end{array}$ & 151 & 56 & $72.94 \%$ \\
\hline $\begin{array}{l}\text { First Four } \\
\text { Moments }\end{array}$ & 151 & 56 & $72.94 \%$ \\
\hline $\begin{array}{l}\text { Statistical } \\
\text { Feature set }\end{array}$ & 151 & 56 & $72.94 \%$ \\
\hline $\begin{array}{l}\text { Region } \\
\text { Properties }\end{array}$ & 155 & 52 & $74.87 \%$ \\
\hline
\end{tabular}

The clinical symptoms play important role in the diagnosis of Osteoarthritis. The investigation of the disease is carried out by both clinical symptoms and radiological assessment. As we have considered only the radiological assessment of knee Xray, the miss classification rate is bit high as mentioned in above table. Since the result obtained from above table-2 seems to be less informative in classifying the image, we experimented on merging the feature set. Fusion of features provides a combination of relevant information of two or more feature set. The resulting combined feature set can be more instructive than individual feature sets. The table-3 below gives the classification rate of merged feature set. 
Table-3 Classification rate of merged feature set

\begin{tabular}{|c|c|c|c|}
\hline $\begin{array}{c}\text { Fusion of } \\
\text { Feature Set }\end{array}$ & $\begin{array}{c}\text { Correctly } \\
\text { Classified }\end{array}$ & Misclassified & $\begin{array}{c}\text { Accuracy } \\
\text { rate }\end{array}$ \\
\hline $\begin{array}{c}\text { (Texture+ } \\
\text { Haralick + First }\end{array}$ & 182 & 25 & $87.92 \%$ \\
$\begin{array}{c}\text { Four Moments } \\
\text { Statistical + } \\
\text { Region } \\
\text { Properties) }\end{array}$ & & & \\
\hline
\end{tabular}

The graphical representation of all the feature set is shown in the figure 6 . The bar graph clearly demonstrates that the fusion of all the feature set gives better results than the individual feature set.

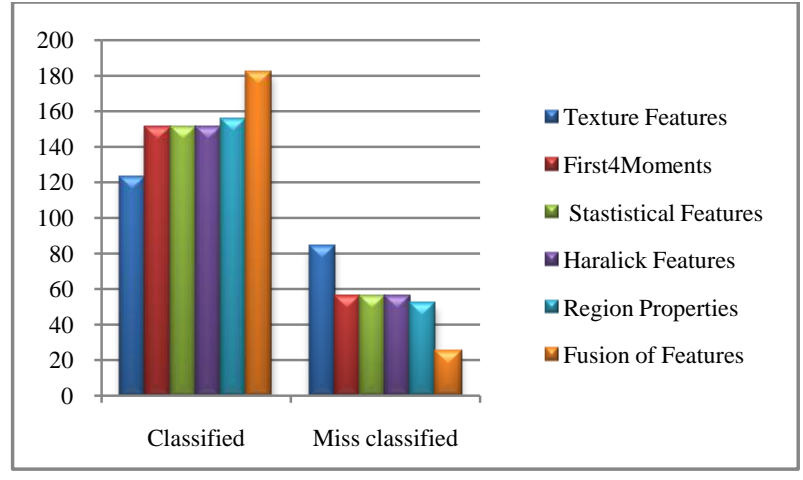

Fig 6.Graphical Representation of Features

Table-4 Confusion Matrix of individual feature set

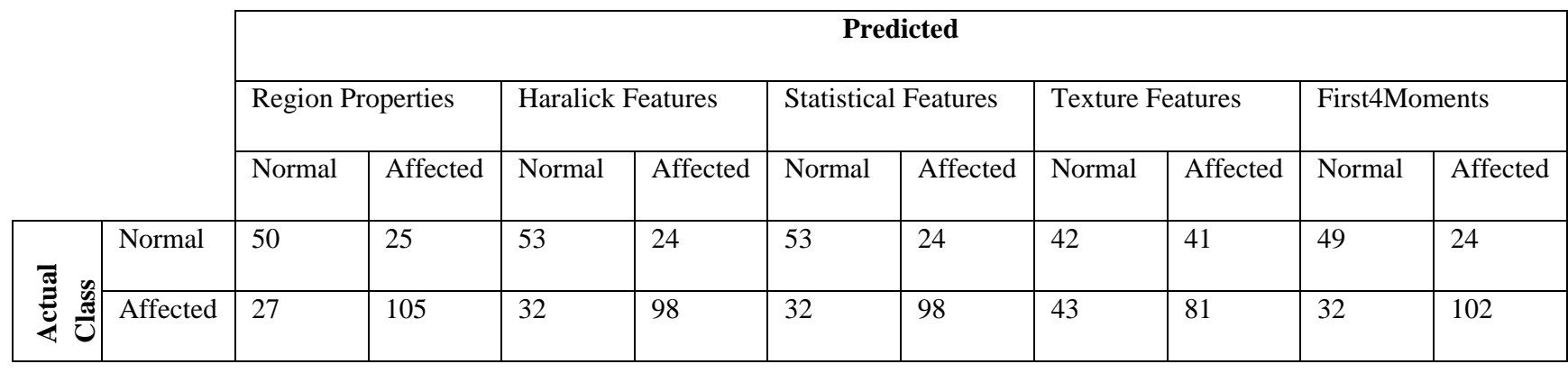

\section{CONCLUSION}

Analysis of X-ray images is done manually by the physician that is time consuming, subjective \& unpredictable. The complexities associated with the X-ray images make it difficult to analyze them in an effective way. A knee X-ray image is very much prone to unwanted distortions that cause problem in analyzing the bone structures. To overcome these problems authors have used semi-automated technique that provides a quick and efficient method to analyze the abnormalities \& problems associated with the bone structures. In the work authors have used Active contour algorithm to segment a knee x-ray image that undergoes various feature extraction techniques. The extracted features demonstrated the accuracy rate of $\mathbf{8 7 . 9 2 \%}$ using Random Forest classifier. In future the technology or process need to be developed that is associated to Osteoarthritis pain and clinical symptoms, such as whether the symptoms are related to joint tissue, neuropathic pain, muscular pain etc. This may help in acquiring good classification rate.

\section{ACKNOWLEDGMENTS}

Authors would like to thank Dr. Kiran S.Marathe M.S(Ortho) $_{\text {, }}$

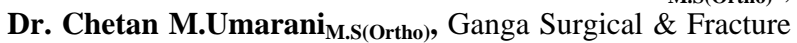
Clinic ,Gokak-Belagavi-Karnataka India, Dr Manjunath V.Shindholimath M.S(Ortho), Vachanamruta Hospital, Gokak Belagavi-Karnataka India and Dr. P S Hiremath, KLE Technological University, Hubli-Karnataka India for providing knee $\mathrm{x}$-ray images \& useful information related to the analysis of Osteoarthritis.

\section{REFERENCES}

[1] Lior Shamir, Shari M.Ling,William W.Scott, Angelo Bos, Nikita Orlov, Tomasz Macura, D.Mark, Luigi Ferrucci, Ilya G.Goldberg "Knee X-ray Image Analysis Method for Automated Detection of Osteoarthritis", IEEE Transactions on Biomedical Engineering, (C2008.
[2] Samir K.Bandyopadhyay "An Edge Detection Algorithm for Human Knee Osteoarthritis Images", Journal of Global Research in Computer Science, Volume 2, No. 4, ISSN-2229-371X, April 2011.

[3] Dipali D.Deokar, Chandrashekar G.Patil "Effective feature Extraction Based Automatic knee Osteoarthritis Detection and Classification using Neural Network", International Journal of Engineering and Techniques, ISSN:2395-1303, Volume 3, Issue 3, PP: 134-139, May 2015

[4] M. Subramoniam and V. Rajini "Local Binary Pattern Approach to the Classification of Osteoarthritis in Knee X-ray Images", Asian Journal of Scientific Research, Volume 6, Issue 4, pp: 805-811, ISSN: 1992-1454, 2013.

[5] Prafull Sharma, Joshua Madhukar Singh " A Novel Approach towards X-ray Bone Image Segmentation using Discrete Step Algorithm", International Journal of Emerging Trends \& Technology in Computer Science, Volume 2, Issue 5, PP:191-195, ISSN 2278-6856, 2013.

[6] Bindushree R, Sanjeev Kubakaddi, Nataraj Urs “ Detection of Knee Osteoarthritis by Measuring the Joint Space Width in Knee X ray Images", International Journal of Electronics \& Communication, ISSN 23215984, Volume 3, Issue 4, PP:18-21,April 2015.

[7] Dian Pratiwi, Diaz D.Santika, Bens Pardamean "An Application of Back propagation Artificial Neural Network Method for Measuring the Severity of Osteoarthritis", International Journal of Engineering \& Technology, Volume 11, No.3, June 2011.

[8] Subromoniam M, Barani S \& Rajini V "A non-invasive computer aided diagnosis of osteoarthritis from digital X-ray images”, Biomedical Research, ISSN0970-938X, Volume 26, Issue 4, pp:721-729, 2015. 
[9] Lilik Anifah, Ketut Eddy Purnama, Moch Hariadi, Mauridhi Hery Purnomo "Automatic Segmentation of Impaired Joint Space Area for Osteoarthritis Knee X-ray Image using Gabor Filter based Morphology Process", IPTEK, The Journal of Technology \& Science, Volume 22,Issue 3, pp:159-165, August 2011.

[10] Mahima Shanker Pandey, Rajitha B,Suneeta Agarwal “ Computer Assisted Automated Detection of Knee Osteoarthritis using X-ray Images", Science\& technology The International Quarterly Journal, ISSN 2394-3769, Volume 1, Issue 2,PP:74-79, ,April 2015.

[11] Jessie Thomson, Terence O’Neill, David Felson, Tim Cootes "Automated Shape \& Texture Analysis for Detection of Osteoarthritis from Radiographs of the Knee”, Springer International Publishing Switzerland, pp: 127-134, 2015.

[12] Lilik Anifah, Ketut Eddy Purnama, Mochamad Hariadi, Mauridhi Hery Purnomo " Osteoarthritis Classification Using Self Organizing Map Based on Gabor Kernel \& Contrast-Limited adaptive Histogram Equalization", The Open Biomedical Engineering Journal,2013.

[13] Tati L.Mengko,Rachmat G, Wachjudi, Andriyan B.Suksmono, Dony Danudirdjo "Automated Detection of Unimpaired Joint Space for Knee Osteoarthritis Assessment”, @2005 IEEE.

[14] Lior Shamir "A Computer analysis method for correlating knee X-ray with continuous indicators", International Journal of Computer Assisted Radiology \& Surgery (CARS), Volume 6, pp: 699-704, 2011.

[15] Subramonium.M \& Rajini.V "Support Vector Machine Approach for the Diagnosis of Arthritis from Digital Xray Images Using Local Ternary Pattern", Indian Journal of Applied Research, Volume 3, Issue 9, Sept 2013.

[16] Chhanda Ray, Krishnendu Sasma "A New Approach for Clustering of X-ray Images", IJCSI International Journal of Computer Science Issues, ISSN: 1694-0784, Vol. 7, Issue 4, No 8, July 2010.

[17] G.Anil Kumar, NistalaV.E.S.Murthy "Analysis of Medical Image Processing and its Applications in Healthcare Industry", International Journal Computer Technology \& Applications, ISSN: 2229-6093, Volume 5 Issue 3, pp: 851-860, 2014

[18] Ying Shen and Weihua Zhu " Medical Image Processing using A Machine Vision-based Approach", International Journal of Signal Processing, Image Processing and Pattern Recognition Vol. 6, No. 3, June, 2013.

[19] J. E. Dacre And E. C. Huskisson "The Automatic Assessment Of Knee Radiographs In Osteoarthritis Using Digital Image Analysis", British Journal of Rheumatology, Volume 28, pp: 506-510, 1989.

[20] Critina Stolojescu Crisan, Stefan Holban "An Interactive $\mathrm{X}$-Ray Image Segmentation Technique for Bone Extraction", International Work-Conference on Bioinformatics \& Biomedical Engineering (IWBBIO), Volume 2, 2014

[21] Bhagyashri L.Wagaj, M.M.Patil "Osteoarthritis Disease Detection with the help of Image processing technique", International Journal of Computer Applications (09758887), Conference on Emerging Applications of
Electronic System, Signal Processing and Computing Technologies 2015.

[22] Flavia Cicuttini, Andrew Forbes, Kevin Morris, Sandy Darling, Michael Bailey \& Stephen Stuckey "Gender differences in knee cartilage volume as measured by magnetic resonance imaging", Osteoarthritis and Cartilage, volume7, pp: 265-271, 1999.

[23] Bindushree R, Sanjeev Kubakaddi, Nataraj Urs “ Detection of Knee Osteoarthritis by Measuring the Joint Space Width in Knee X ray Images", International Journal of Electronics \& Communication, ISSN 23215984, Volume 3, Issue 4, pp:18-21,April 2015.

[24] M.S.Mallikarjuna Swamy, Mallikarjun S.Holi "Knee Joint Articular Cartilage Segmentation, Visualization and Quantification using Image Processing Techniques", International Journal of Computer Applications (09758887), Volume 42, No.19, pp: 36-41, March 2012.

[25] Til Aach , Ulrich Schiebel and Gerhard Spekowius "Digital Image Acquisition and Processing in Medical XRay Imaging", Journal of Electronic Imaging, Institute of Imaging \& Computer Vision, ISSN 1017-9909 ,Volume 8, Issue 1, pp: 7-22,1999.

[26] G.Behiels, D. Vandermeulen, F.Maes, P.seutens, P.Dewaele "Active Shape Model Based Segmentation of Digital X-ray Images", Second International Conference, Cambridge, UK, September 19-22, ISBN- 978-3-54066503-8, Volume 1679, pp: 128-137, 1999.

[27] T. F. Chan, L. A. Vese, "Active contours without edges" IEEE Transactions on Image Processing, Volume 10, Issue 2, pp. 266-277, 2001.

[28] V. Caselles, R. Kimmel, G. Sapiro, "Geodesic active contours", International Journal on Computer Vision, Volume 22, Issue 1, pp. 61-79, 1997.

[29] Yang Mingqiang, Kpalma Kidiyo and Ronsin Joseph "A Survey of Shape Feature Extraction Techniques", Pattern Recognition Techniques, Technology and Applications, Book edited by: Peng-Yeng Yin, ISBN 978-953-761924-4, pp. 626, November 2008.

[30] B.Jyothi, Y.Madhavee Latha, P.G.Krishna Mohan, V.S.K.Reddy "Medical Image Retrieval Using Moments", International Journal of Application or Innovation in Engineering \& Management (IJAIEM), ISSN 2319 - 4847, Volume 2, Issue 1, January 2013.

[31] Alice Porebskil, Nicolas Vandenbroucke \& Ludovic Macaire "Haralick feature extraction from LBP images for color texture classification”,@2008 IEEE.

[32] Leo Breiman "RandomForests" Machine Learning, Volume: 45, pp: 5-32, 2001 (C) 2001 Kluwer Academic Publishers. Manufactured in The Netherlands.

[33] B.S. Manjunathi and W.Y. Ma "Texture Features for Browsing and Retrieval of Image Data", IEEE Transactions on Pattern Analysis and Machine Intelligence, Vol. 18, NO 8, 1996.

[34] Shivanand S. Gornale, Pooja U. Patravali, Ramesh R. Manza "A Survey on Exploration and Classification of Osteoarthritis Using Image Processing Techniques", International Journal of Scientific and Engineering Research (IJSER), ISSN 2229-5518, Volume 7, Issue 6,July 2016 (Accepted). 